\title{
Non-Organic Failure to Thrive
}

National Cancer Institute

\section{Source}

National Cancer Institute. Non-Organic Failure to Thrive. NCI Thesaurus. Code C128740.

Failure to thrive caused by inadequate intake due to environmental influences, stimulus deprivation- poverty, feeding techniques, or psychological reason, and without any apparent growth-inhibiting organic disorder. 